\title{
Management of Iris Retraction Syndrome with Heterochromia and Retinal Detachment
}

\author{
Collin Goebel Gordon S. Crabtree Michael M. Altaweel \\ Department of Ophthalmology and Visual Sciences, University of Wisconsin School of \\ Medicine and Public Health, Madison, WI, USA
}

\section{Keywords}

Iris retraction syndrome $\cdot$ Retinal detachment $\cdot$ Heterochromia

\begin{abstract}
Iris retraction syndrome (IRS) is an uncommon condition caused by retinal detachment that is characterized by back bowing of the peripheral iris, leading to a deep anterior chamber. It is commonly associated with ocular hypotony, ciliochoroidal detachment, and anterior proliferative vitreoretinopathy. We describe a case of a 66 -year-old man presenting with 2 weeks of right eye pain, redness, and iris heterochromia. The patient was diagnosed with IRS secondary to a chronic retinal detachment. Initial management with topical steroids and mydriasis allowed resolution of the iris retraction and heterochromia, normalization of intraocular pressure, and improvement of choroidal detachment. Subsequent vitrectomy with endolaser and oil tamponade led to successful detachment repair. Initial pharmacologic management allows a more controlled approach to the repair of retinal detachment associated with IRS. The patient's presentation is consistent with the hydrodynamic hypothesis of IRS.
\end{abstract}

(C) 2021 The Author(s).

Published by S. Karger AG, Basel

\section{Introduction}

Iris retraction syndrome (IRS) is a rare condition characterized by chronic retinal detachment, hypotony, and uveitis, leading to adherence of the iris to the anterior lens with resulting pupillary block. This results in back bowing of the iris in a configuration opposite to 
iris bombé. Concurrent inflammation may lead to iris heterochromia due to an increase in yellow proteinaceous fluid in the anterior chamber.

The condition was first described by Schnabel [1] in patients with retinal detachment, hypotony, and deep anterior chambers. Several years later, Leber [2] noted inflammation as a key component of IRS. Other reports described increased risk for iris retraction and poor surgical prognosis in cases of retinal detachment with associated ciliochoroidal detachment [3, 4]. In 1984, Campbell [5] was the first to name the condition IRS and proposed the most commonly accepted mechanism for the condition, in which seclusion of the pupil and egress of subretinal fluid are the drivers of iris retraction. However, debate remains regarding the mechanism of fluid egress that leads to hypotony, as Geyer et al. [6] reported 2 cases of IRS associated with nonrhegmatogenous retinal detachment. In 1996, Morinelli et al. [7] demonstrated with ultrasonography the characteristic anterior chamber appearance of IRS. The case of IRS associated with rhegmatogenous retinal detachment described here demonstrates the importance of managing the uveitic and pupillary block components that contribute to IRS prior to or contiguous with surgical repair.

\section{Case Report}

A 66-year-old man presented with 2 weeks of right eye pain, redness, and a nasal visual field defect. Past ocular history was significant for cataract surgery in the left eye. He had no history of previous trauma and was systemically healthy. Examination revealed a visual acuity of $20 / 400$, ciliary flush, and iris heterochromia with a green right iris compared to the blue left iris (shown in Fig. 1). Posterior synechiae were present $360^{\circ}$ in the right eye. Intraocular pressure (IOP) was $6 \mathrm{~mm} \mathrm{Hg}$ in the right and $17 \mathrm{~mm} \mathrm{Hg}$ in the left. Gonioscopy and slit-lamp examination demonstrated a very deep right anterior chamber with inferior posterior synechiae, 2+ flare, and no cell. Ultrasound biomicroscopy (UBM) demonstrated a posterior bowing of the iris and diffusely deepened anterior chamber (shown in Fig. 2). Fundus examination demonstrated a rhegmatogenous retinal detachment and choroidal detachment in the right eye and a normal fundus appearance in the left eye.

The constellation of findings was consistent with IRS. Choroidal detachment with a hazy view would have made the repair of the retinal detachment more difficult; therefore, treatment with difluprednate $0.05 \%$ qid and atropine $1 \%$ bid was initiated to reduce intraocular inflammation and attempt to reverse the pupillary block. At 7-day follow-up, the conjunctival injection and vitreous haze in the right eye were decreased, and the iris approached its baseline blue color (shown in Fig. 3). The IOP was increased to $17 \mathrm{~mm} \mathrm{Hg}$, compared to $6 \mathrm{~mm}$ Hg at presentation. UBM showed resolution of iris retraction with normal anterior chamber depth (Fig. 2), broken synechiae, and resolved anterior chamber reaction. The choroidal detachment had improved significantly. Three weeks following the initiation of steroid and atropine drops, the patient underwent surgery with 25-gauge pars plana vitrectomy, endolaser, and 1,000 cc silicone oil tamponade to manage inferior proliferative vitreoretinopathy (PVR), with successful attachment. Silicone oil removal and cataract surgery were performed 3 months after vitrectomy, and laser peripheral iridotomy was required for an episode of angle closure glaucoma 2 months later. The visual acuity stabilized at 20/200, measured 2 years after laser peripheral iridotomy.

\section{Discussion}

IRS is a rare condition that is associated with chronicinflammation and retinal detachment. Posterior synechiae and egress of fluid from the posterior segment combine to create the unique morphology of iris retraction, with a very deep anterior chamber and back bowing of

\section{Karger'}




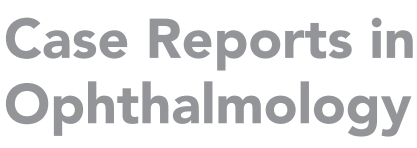

Case Rep Ophthalmol 2021;12:809-815

\begin{tabular}{l|l}
\hline DOI: $10.1159 / 000519148$ & (c) 2021 The Author(s). Published by S. Karger AG, Basel
\end{tabular} www.karger.com/cop

Goebel et al.: Iris Retraction Syndrome
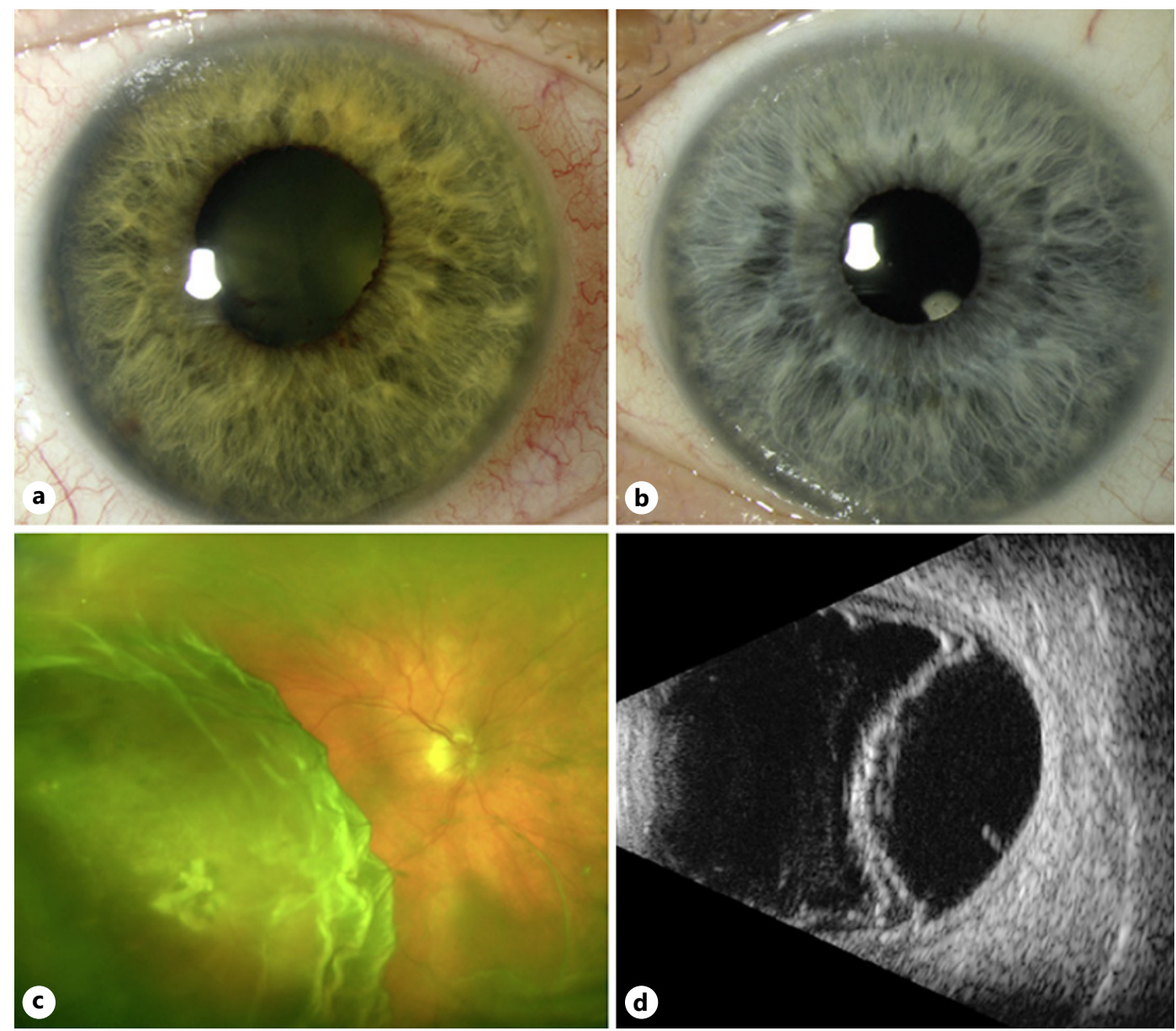

Fig. 1. Initial presentation. a External photograph of the patient's right eye demonstrating green discoloration, ectropion uvea, and conjunctival injection. b External photograph of the patient's left eye. c Fundus photograph displaying a rhegmatogenous retinal detachment and choroidal detachment in the right eye. d Ultrasound image demonstrating the retinal and serous choroidal detachment.

the iris [8]. PVR is associated with IRS, hypothesized to be a result of concomitant uveitis, chronic retinal detachment, and choroidal detachment that allow influx of protein and cells into the vitreous cavity.

Green discoloration of the iris has been noted in several previous cases of IRS [3]. This discoloration is a result of proteinaceous fluid that accumulates in the anterior chamber following chronic retinal detachment, uveitis, and pupillary block. After treatment of iris retraction and the return of normal aqueous flow, the fluid in the anterior chamber is cleared through the trabecular meshwork, and the discoloration of the iris resolves. In the case of our patient, the heterochromia normalized following pharmacologic resolution of pupillary block, even before vitrectomy surgery.

Repair of retinal detachment in cases of IRS has typically been with vitrectomy, endolaser, and gas or silicone oil tamponade. As choroidal detachment and PVR are common with the concurrent uveitis, repair may be more complicated, and prognosis is more guarded. This patient underwent surgery with a 25-gauge pars plana vitrectomy with a posterior drainage retinotomy, endolaser, and 1,000 cc silicone oil placement due to the finding of inferior PVR and residual choroidal detachment. The success rate of retinal detachment repair is greater when choroidal detachment is absent. Pharmacologic management of IRS with mydriasis to 


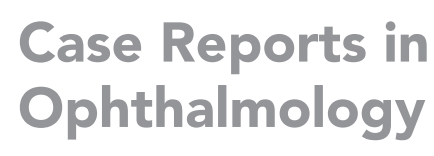

Case Rep Ophthalmol 2021;12:809-815

\begin{tabular}{l|l}
\hline DOI: $10.1159 / 000519148$ & ○ 2021 The Author(s). Published by S. Karger AG, Basel
\end{tabular} www.karger.com/cop

Goebel et al.: Iris Retraction Syndrome

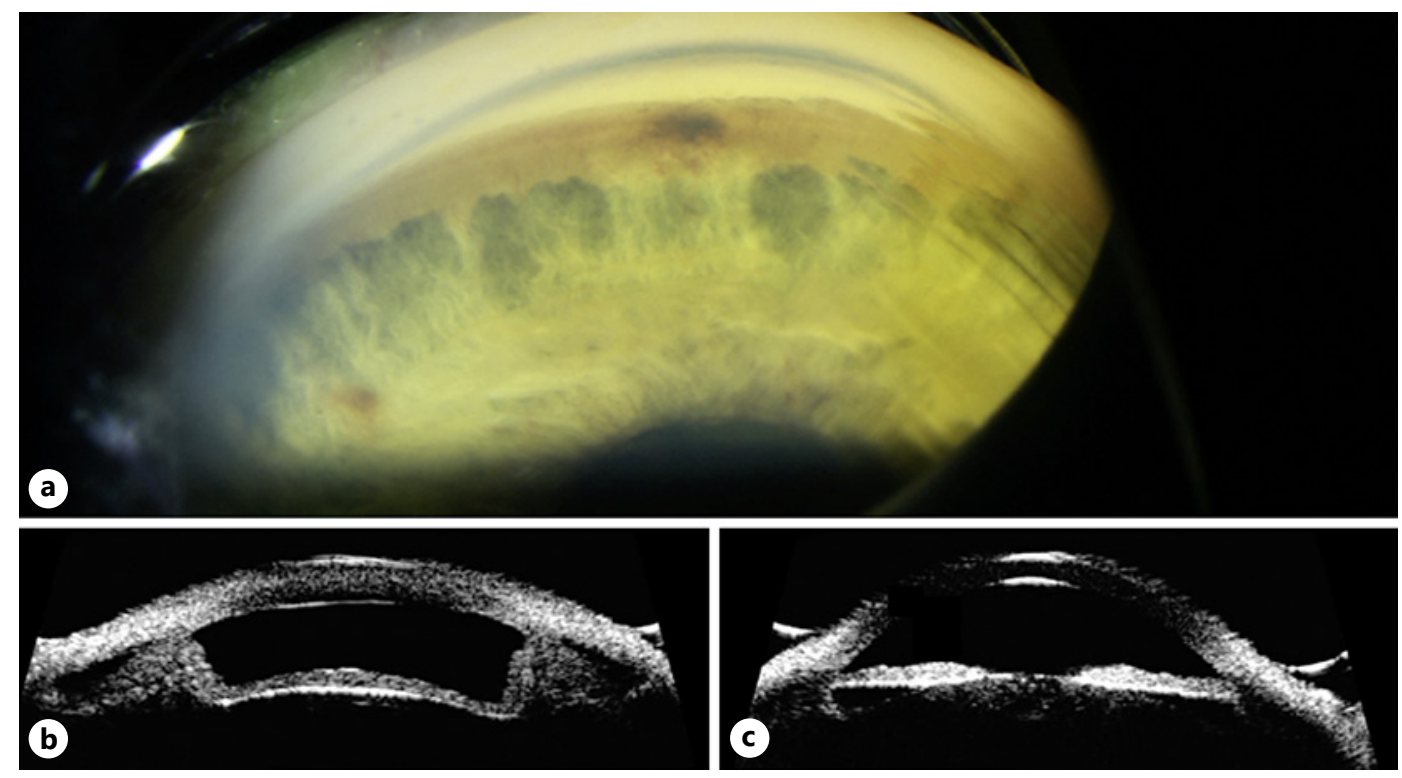

Fig. 2. Anterior chamber findings. a Gonioscopy image of the right eye at initial presentation displaying markedly widened anterior chamber. b Initial UBM of patient's right eye demonstrating peripheral back bowing of the iris and widening of the anterior chamber. c UBM of patient's right eye following resolution of iris retraction and normalization of anterior chamber depth. UBM, ultrasound biomicroscopy.

break the pupillary block and topical or regional steroids to manage the uveitis can allow normalization of IOP, reduction of choroidal detachment, and normalization of anterior chamber depth. Seelenfreund et al. [8] found that $81.5 \%$ of 50 cases with RD and choroidal detachment had improved their effusion with preoperative steroid treatment. These anatomical changes allow safer entry of sclerotomy blades for vitrectomy, stabilize intraocular hydrodynamics, and improve the intraoperative visualization of retinal breaks and effectiveness of laser.

The differential diagnosis of IRS includes angle recession glaucoma, pigment dispersion syndrome, Fuchs heterochromatic iridocyclitis, postsurgical anterior chamber deepening, and uveitis. Uveitis with cyclitic membrane can cause chronic hypotony due to ciliary body shutdown. Although pigment dispersion syndrome can rarely cause a similar iris configuration to IRS, this is associated with spikes of increased IOP, rather than the hypotony present in IRS.

The most widely accepted mechanism of IRS was described by Campbell [5]. Prior to Campbell, researchers hypothesized that IRS may occur due to vitreous retraction or lack of aqueous humor production $[9,10]$. Campbell was the first to suggest seclusion of the pupil as essential to the mechanism. In this model, due to a rhegmatogenous retinal detachment and seclusion of the pupil, aqueous humor is pumped out of the eye after passing through the retinal defect. If this efflux exceeds the production rate of the ciliary body, there is a resulting decrease in IOP and a posterior displacement of the iris. Campbell provided evidence for his hypothesis with 2 cases of IRS following carbonic anhydrase inhibitor administration for iris bombé. The ensuing decrease in production of aqueous humor, in the presence of secluded pupil and retinal detachment, shifted the equilibrium toward excess aqueous humor removal after passing through the retinal defect. The change in pressure dynamics resulted in posterior displacement of the iris. Our patient's findings of chronic retinal and choroidal detachment in association with iris retraction, uveitis, and hypotony are all consistent with Campbell's model of IRS, as is the improvement that was noted with release of pupillary block and management of uveitis before surgical repair. 


\section{Case Reports in Ophthalmology}
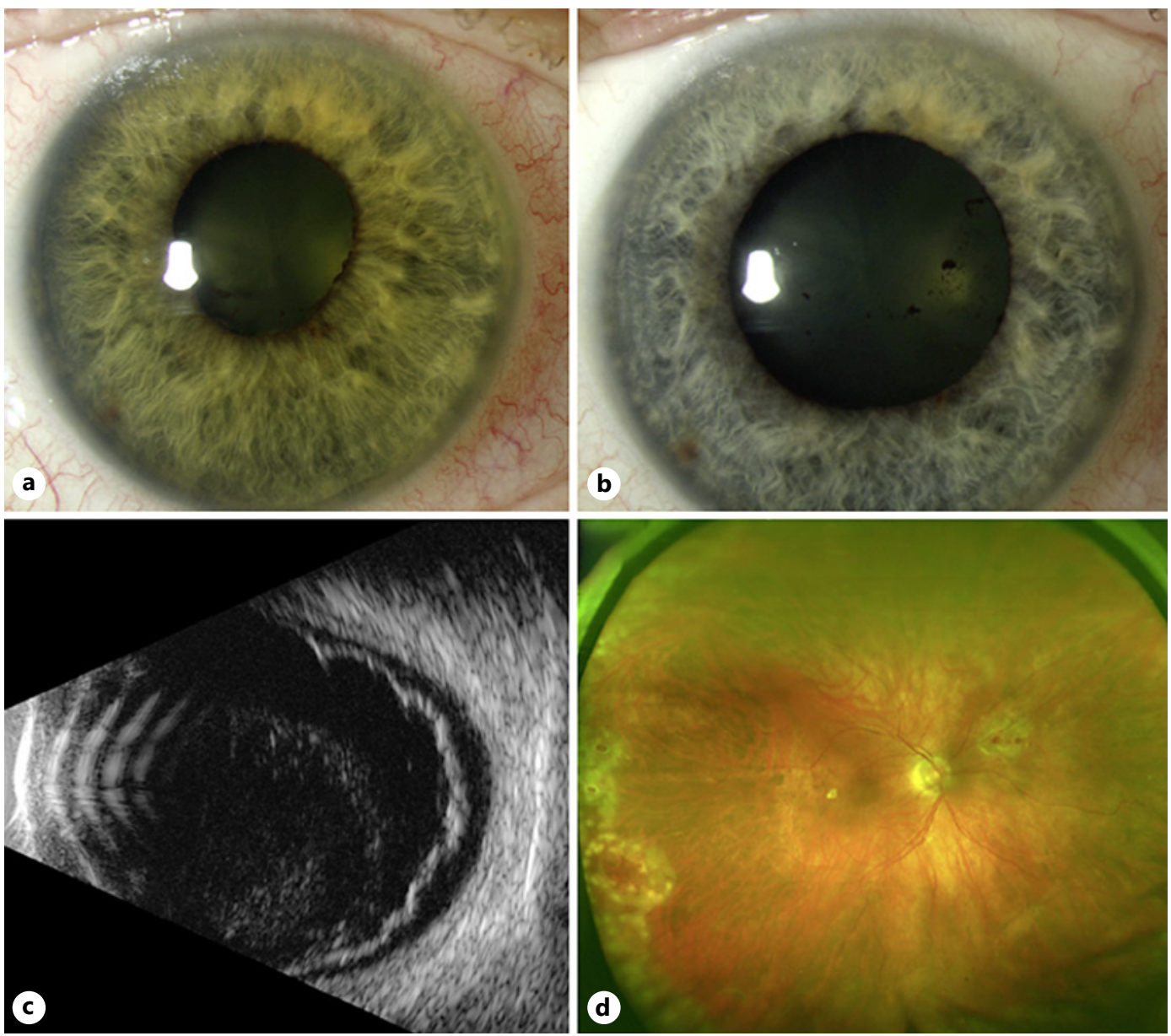

Fig. 3. Following treatment. a External photograph of the patient's right eye at initial presentation. b External photograph of the patient's right eye 1 week after initiation of difluprednate and atropine drops. Resolution of green discoloration can be observed. c Ultrasound image of the right eye after resolution of hypotony and serous choroidal detachment 1 week following drop initiation; retinal detachment remains. d Fundus photograph 1 week following retinal detachment repair with vitrectomy, endolaser, and silicone oil placement.

The prognosis of IRS is variable and depends on the extent of PVR, choroidal detachment, and uveitis. Previous cases have shown that PVR is an indicator of poor visual acuity outcome following treatment $[3,8]$. Our patient's visual acuity remained 20/200, despite successful retinal detachment repair and management of iris retraction. Campbell demonstrated how iris retraction and iris bombé can be associated, by noting the conversion to iris retraction with the administration of aqueous suppressants to his patients with iris bombé and return to iris bombé again when the medication was stopped [6]. Given this, laser peripheral iridotomy should be considered in patients with IRS to prevent subsequent episodes of angle closure glaucoma, as occurred in our patient.

\section{Conclusion}

IRS is a rare condition associated with back bowing of the iris, pupillary block, uveitis, and heterochromia that should be considered in patients with rhegmatogenous retinal detachment. Gonioscopy and UBM are useful tests in making the diagnosis. Pharmacologic management 
with steroids and mydriasis can alleviate the iris retraction and may improve the surgical outcome and ease of retinal detachment repair.

\section{Statement of Ethics}

This case report was conducted according to good clinical practice. The authors state that they have full control over all primary data and have no ethical conflicts to disclose. This study protocol was reviewed, and the need for approval was waived by the IRB at the University of Wisconsin-Madison. The patient provided written consent for these data and images to be published.

\section{Conflict of Interest Statement}

The authors have no financial disclosures and no commercial or proprietary interest in any materials discussed in this report.

\section{Funding Sources}

Funding for this research was provided by an Unrestricted Grant from Research to Prevent Blindness, Inc., to the University of Wisconsin Department of Ophthalmology and Visual Sciences, National Eye Institute Grant U10EY014656, and the McPherson Eye Research Institute Monroe E. Trout Chair in Vision research at the University of Wisconsin (Dr. M.M. Altaweel).

\section{Author Contributions}

Collin Goebel participated in the acquisition of data, interpretation of data, drafting of the manuscript, and critical revising of the manuscript. Gordon S. Crabtree participated in the acquisition of data, interpretation of data, and drafting of the manuscript. Michael M. Altaweel participated in the acquisition of data, interpretation of data, and drafting of the manuscript. All authors provided final approval of the manuscript version to be published.

\section{Data Availability Statement}

All data generated or analyzed during this study are included in this article. Further enquiries can be directed to the corresponding author.

\section{References}

1 Schnabel J. Ueber glaukom und iridectomie. Arch Augenheilkd. 1876;5:50-89.

2 Leber Th. Die krankheiten der netzhaut. In: Saemisch Th, Elschnig A, editors. Graefe-saemisch-hess handbuch der gesamten augenheilkunde, Vol. 7A. Leipzig: W. Engelmann; 1916. p. 1422-8.

3 Gottlieb F. Combined choroidal and retinal detachment. Arch Ophthalmol. 1972;88(5):481-6.

4 Jarrett WH. Rhematogenous retinal detachment complicated by severe intraocular inflammation, hypotony, and choroidal detachment. Trans Am Ophthalmol Soc. 1981;79:664-83.

5 Campbell DG. Iris retraction associated with rhegmatogenous retinal detachment syndrome and hypotony. A new explanation. Arch Ophthalmol. 1984;102(10):1457-63.

\section{Karger's}


6 Geyer 0, Neudorfer M, Rothkoff L, Michaeli-Cohen A, Lazar M. Iris retraction syndrome associated with nonrhegmatogenous retinal detachment. Acta Ophthalmol Scand. 1998;76(5):617-9.

7 Morinelli EN, Gentile R, Stegman Z, Liebmann JM, Shabto U, Ritch R. Ultrasound biomicroscopic features of iris retraction syndrome. Ophthalmic Surg Lasers. 1996;27(4):257-62.

8 Seelenfreund MH, Kraushar MF, Schepens CL, Freilich DB. Choroidal detachment associated with primary retinal detachment. Arch Ophthalmol. 1974;91(4):254-8.

9 Gonin J. La pathogénie du décollement spontané de la rétine. Ann Oculist. 1904;132:30-55.

10 Pederson JE. Experimental retinal detachment. IV. Aqueous humor dynamics in rhegmatogenous detachments. Arch Ophthalmol. 1982;100(11):1814-6. 